\title{
Comparison of Prompting Hierarchies on the Acquisition of Leisure and Vocational Skills
}

\author{
Julie A. McKay • Julie S. Weiss • Chata A. Dickson • \\ William H. Ahearn
}

Published online: 4 September 2014

(C) Association for Behavior Analysis International 2014

Task analyses are used for teaching response chains, perhaps most notably to individuals with intellectual disability and/or autism spectrum disorders. Selection of effective tactics for prompting desired responses within a response chain and subsequently fading these prompts is critical to the effectiveness of task analysis as a teaching strategy.

For a response chain to be useful to the learner, responding must eventually occur in the presence of natural stimuli and be maintained by natural consequences. Thus, stimulus control should be transferred from the prompt to the naturally occurring stimuli. This is often achieved using a prompting hierarchy (Billingsley and Romer 1983). It is widely accepted that prompts should be faded so that the response is occasioned by natural cues. Wolery and Gast (1984) defined four procedures for transferring stimulus control from response prompts to natural stimuli. These procedures are most-to-least fading of prompts (MTL), least-to-most fading of prompts (LTM), graduated guidance, and time delay. Generally, MTL and LTM prompting hierarchies, with or without a time delay, are used to fade physical prompts. MTL fading involves gradually reducing the amount of physical assistance provided as training progresses from trial to trial and session to session. LTM fading involves giving the participant an opportunity to perform the response with the least restrictive prompt on each trial. The participant receives greater degrees of assistance until a correct response is emitted (Libby et al. 2008).

J. A. McKay $\cdot$ J. S. Weiss $(\bowtie) \cdot$ C. A. Dickson $\cdot$ W. H. Ahearn

The New England Center for Children, 33 Turnpike Road,

Southborough, MA 01772, USA

e-mail: jweiss@necc.org

W. H. Ahearn

e-mail: bahearn@necc.org

J. A. McKay · J. S. Weiss • C. A. Dickson • W. H. Ahearn

Northeastern University, Boston, MA, USA
At this time, there is not a consistent body of evidence upon which to base selection of prompting and prompt fading strategies. Systematic study of various prompting hierarchies is, therefore, an important endeavor, as more evidence is needed for aiding teachers and behavior analysts as they choose between instructional strategies (Spooner and Spooner 1984). Some initial generalizations, however, have been deduced from the existing literature. Demchak (1990) reviewed several articles that compared effects of various prompt fading strategies on rate of acquisition and found MTL prompting generally to be more efficient than LTM. More recently, Libby et al. (2008) compared MTL and LTM prompting on the acquisition of solitary play skills with five children with autism spectrum disorders. All participants learned to build play structures with MTL, whereas only three of the five learned to build play structures with LTM. Additionally, MTL was associated with fewer errors, and there is much support in the published literature for minimizing the number of errors a learner encounters (Carr and Durand 1985; Demchak 1990; Etzel and LeBlanc 1979). Finally, the three students for whom LTM was effective acquired skills more quickly with LTM than with MTL. It is possible that for these three learners the rate of acquisition was limited by the MTL prompt fading procedure, which required several correct responses at each prompt level before progressing to the next less intrusive prompt level. In this way, the prompting could have prevented independent responding. To address this issue of limiting the rate of acquisition in MTL, Libby et al. conducted a second experiment in which a constant 2 -s time delay was added to the MTL sequence (MTLD). Response acquisition with MTLD was more rapid than with the MTL sequence without a delay. The investigators concluded that the use of MTLD is the best default prompting hierarchy when the instructor is unable to assess multiple teaching procedures but that the most effective teaching strategy was idiosyncratic. 
When it comes to selecting instructional strategies, it makes sense to choose teaching procedures that lead to task mastery the quickest (Demchak 1990). Recent research has investigated more effective ways to teach behavior chains (e.g., Bancroft et al. 2011; Grow et al. 2009; Libby et al. 2008). The effectiveness of an intervention is limited by the factors related to its implementation; these include both precise and consistent implementation over time (Detrich 1999). Even when a teaching strategy is shown to be effective under ideal conditions, its success ultimately will depend on whether they are effective when implemented by the instructors and parents who use them (Allen and Warzak 2000; Axelrod 1991, 1992). Allen and Warzak emphasize the importance of designing and promoting interventions that are as simple as possible, as treatment complexity has been suggested as interfering with treatment integrity and, presumably, learning. In the case of strategies for teaching using task analysis (TA), a lack of precision in the delivery of prescribed prompts is likely to interfere with acquisition (Grow et al. 2009). It may be beneficial to simplify the prompting hierarchy by reducing the number of prompt levels.

As simpler teaching procedures appear to be easier for teachers to learn and consistently implement, these procedures may ultimately have the greatest impact on student performance (Axelrod 1991). The purpose of the current study was to assess the effectiveness and efficiency of two prompting hierarchies, differing in complexity. The goal was to evaluate effectiveness and efficiency of a prompt hierarchy that is simpler and, therefore, that may be easier to implement than MTLD. Rather than more gradually fading physical prompts, as required in MTLD, in manual guidance with 2-s delay (MGD), teachers use only one type of physical prompt: full manual guidance. Participants were taught to build structures with Lego ${ }^{\circledR}$ blocks as they might during solitary leisure activities, similar to Libby et al. (2008). They were also taught to build structures with nuts, bolts, and washers to determine whether the results from the Lego ${ }^{\circledR}$ structure comparison would be replicated with vocational tasks. The prompting hierarchies were compared in the acquisition of these construction tasks.

\section{Method}

\section{Participants and Setting}

Three adolescent girls and one man participated in this study. All participants were diagnosed with an intellectual disability and attended the same school or the affiliated adult services program of the school. The prerequisites for participating in the study were matching colors and a history of acquiring skills with teaching procedures that included physical prompting.

Greta was a 14-year-old adolescent girl diagnosed with Angelman syndrome. Greta displayed severe deficits in expressive language skills and used American Sign Language (ASL) and the Picture Exchange Communication System to communicate. In the 3 years Greta attended the school, she had regularly been exposed to TAs for self-care and acquired the targeted chains when taught with physical prompts. Wendy and Claire were both 15-year-olds diagnosed with pervasive developmental disorder-not otherwise specified. Both Wendy and Claire communicated their wants and needs vocally. Task analyses for self-care, cooking, and leisure were present in both Wendy and Claire's education programs during this study, and both adolescents had extensive past exposure to TAs and readily acquired targeted skills via physical prompting. Greta, Wendy, and Claire all lived in the same residence. Fred was a 37-year-old man diagnosed with an autism spectrum disorder. He displayed severe deficits in expressive language and used American Sign Language and a communication book with words and images to communicate. He also had extensive past exposure to TAs and readily acquired targeted skills via physical prompting. He resided at a residence with other men and women having diagnoses similar to his.

Leisure rooms in the residences and classrooms in the participants' school were used for study. For a given participant, all training sessions occurred in the same setting.

\section{Materials and TAs}

Materials in the training setting were a table, two chairs, data sheets, pencils, and task materials, preferred edibles, and a video camera. Four 12-step structures made from Lego ${ }^{\circledR}$ blocks were used (as in Libby et al. 2008). Each structure consisted of the same size and shape Lego ${ }^{\circledR}$ baseboard and 11 Lego ${ }^{\circledR}$ pieces associated with a specific placement on the baseboard. Two of these baseboards were green, one was yellow, and the other one was red. The two green baseboard structures were not used simultaneously at any point with any participant. Completed structures are shown in Fig. 1. For each structure, the individual building pieces varied in color and shape, but only one was used for each step in the chain. A 12-step TA that specified the order of placement of the pieces was developed for each structure by customizing the Play Skills (Simple Construction) lesson plan from the Autism Curriculum Encyclopedia ${ }^{\circledR}$ (ACE; Mansfield et al. 2011). The first step of the chain was for the participant to pull the base out from a group of the blocks, the second and all following steps involved picking up and placing one block in the order determined by the TA. Two 12-step vocational structures, referred to in this study as chess king and queen pieces, were also used. They both involved the assembly of a screw and bolt with different nuts and washers. Each structure consisted of the same size and shape pieces. A 12-step TA that specified the order of placement of the pieces was also developed for the vocational structures by customizing the Simple Assembly Task lesson plan from the ACE. The first step of the 

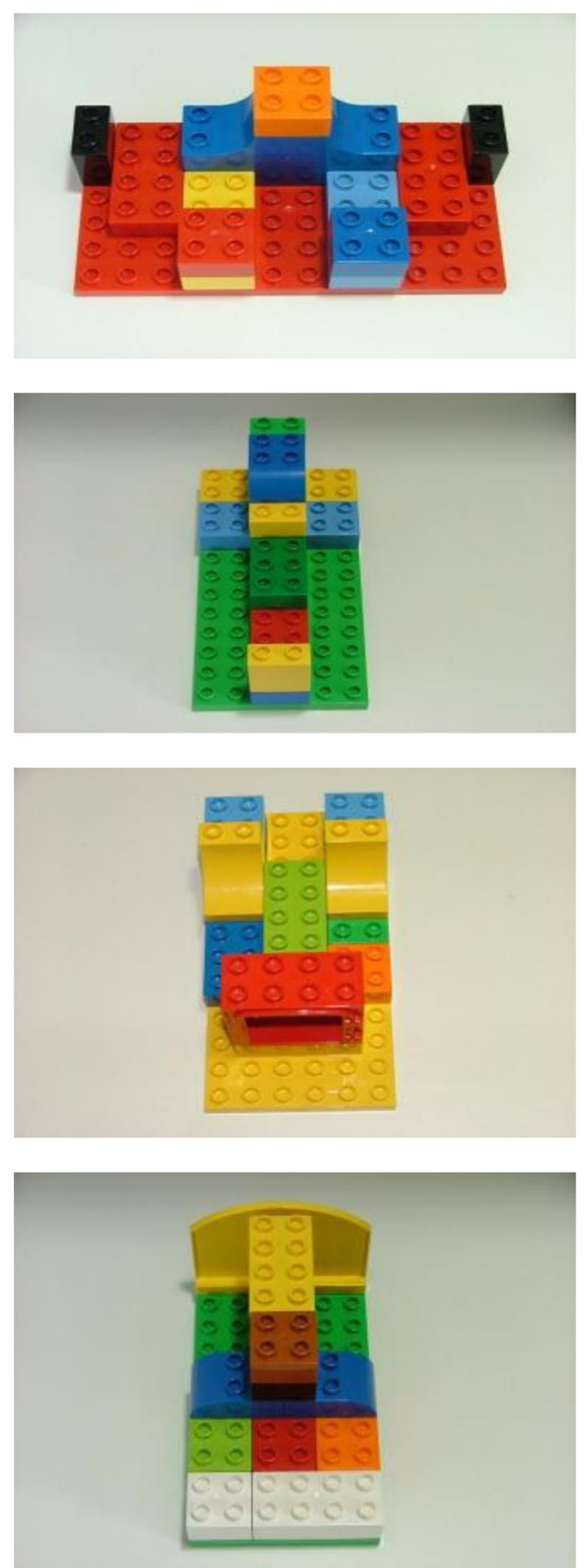

Fig. 1 Four completed Lego ${ }^{\circledR}$ structures with baseboards and different color pieces

chain was for the participant to pick up the screw from a group of pieces. The second and all following steps involved picking up and placing one piece on the screw in the order determined by the TA. Completed vocational structures are shown in Fig. 2. Care was taken to ensure that the four $\operatorname{Lego}^{\circledR}{ }^{\circledR}$ tasks and that the two vocational tasks were of approximately equal difficulty. For each participant, structures were assigned arbitrarily to a teaching condition.
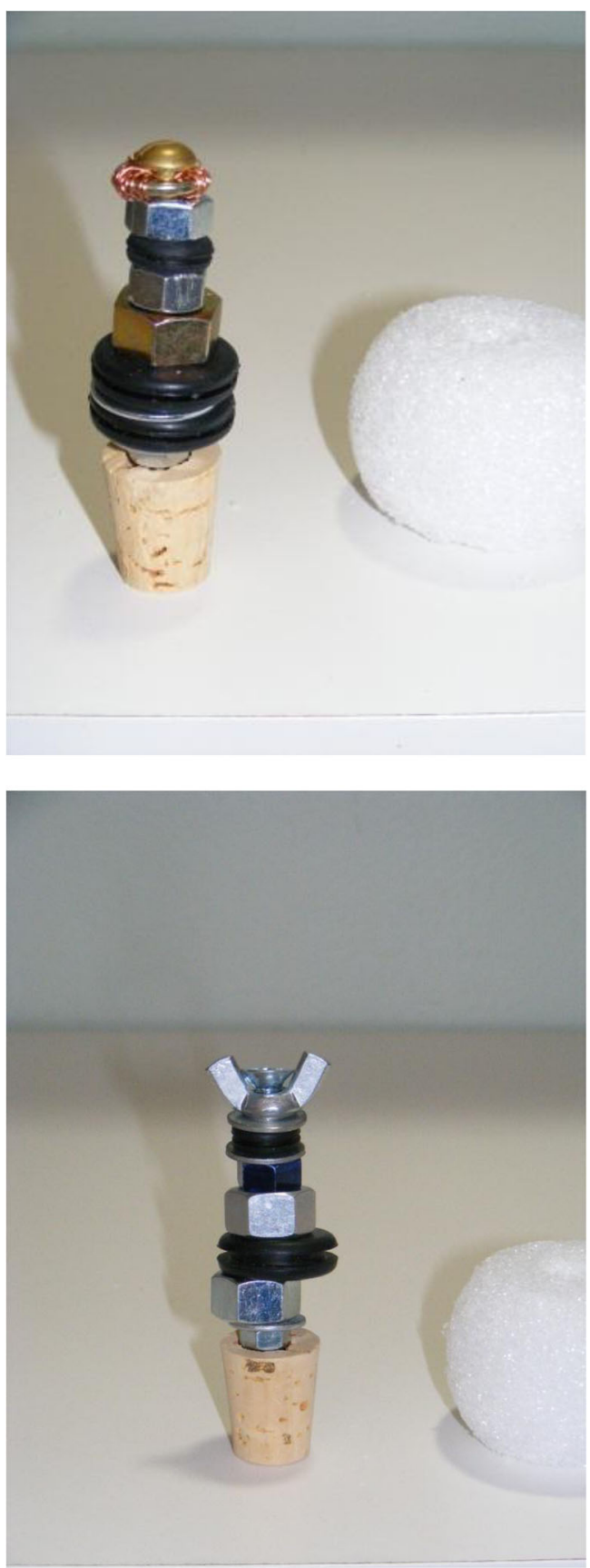

Fig. 2 Two completed vocational structures with screws, nuts, bolts, and washers

\section{Procedure}

Prior to the study, the experimenter conducted preference assessments with edible items for each participant (Fisher et al. 1992). At the beginning of each experimental session, the participant chose between two to three highly preferred edibles and that item was delivered following correct responding during that session.

Forward chaining was used to teach all chains. Each successive step in the chain was taught after the participant had 
mastered the previous step of the chain. Sessions consisted of one probe trial followed by ten training trials. Probe trials served as an opportunity for a participant to respond independently. During probe trials, all of the materials for the task were arrayed in front of the participant and the experimenter established attending. After the initial $S^{\mathrm{D}}$ of "Let's build Legos ${ }^{\circledR !}$ !" or "Let's build a chess piece!", items remained for $15 \mathrm{~s}$ or until the participant made an error. The position of the items varied unsystematically from trial to trial. The experimenter provided no prompting or reinforcement. Probe data from the first session served as baseline. None of the participants demonstrated any correct steps in the chaining sequences during baseline probe trials.

During training trials, response chains were taught either with MTLD or MGD. Task items were arrayed unsystematically in front of the participant and the experimenter established attending. After the initial $\mathrm{S}^{\mathrm{D}}$ of "Let's build Legos ${ }^{\circledR !}$ !" or "Let's build a chess piece!", the experimenter allowed the participant to complete mastered steps independently and followed the predetermined prompting hierarchy for the training step. Correct responses on the training step were followed by a preferred edible item and verbal praise. Incorrect responses on the training step or on previously acquired steps of the chain were corrected immediately with hand-over-hand guidance, and no edible or praise was delivered. Each of the two prompting techniques was associated with a different response chain. Untrained steps were not completed.

The prompting levels associated with each of the prompting hierarchies are shown in Fig. 3. Note that there are five levels of prompting in MTLD, as compared to three prompt levels in MGD. For each step in each response chain, prompt levels within a training step progressed to the next less restrictive level following correct prompted responses on two consecutive trials. Training of the next step in a response chain began following correct independent responses on two consecutive trials. Prompt levels within a step regressed to the next more restrictive level following errors on two consecutive trials. Previously mastered steps were retrained following errors on two consecutive trials. A structure was considered mastered when all steps in a chain were completed independently for two consecutive trials. If performance of a probe trial was $100 \%$ independent and accurate, the experimenter conducted another probe trial. If the participant completed the structure independently again, the structure was mastered. In this way, a structure could be mastered at start of a session based on performance during probe trials. Once a structure was mastered, generalization probes were conducted by a novel teacher in a different room. Generalization was said to have occurred if the entire response chain was completed correctly with the novel teacher and in a different setting. These probes usually were conducted the same day mastery was achieved and were continued with each participant until

\begin{tabular}{|l|}
\hline MTLD \\
\hline Hand over hand \\
2-s delay, manual guidance at upper arm \\
2-s delay, light touch/shadow \\
Independent \\
\hline MGD \\
\hline Hand over hand \\
2-s delay, hand over hand \\
Independent
\end{tabular}

Fig. 3 Prompt levels associated with the two prompting hierarchies. The most intrusive prompt in the sequence is shown in boldface type, and the opportunity for independent performance during training trials is italicized

generalization occurred. On these trials, reinforcement was delivered only following the completion of the entire response chain.

Performance associated with the two different prompting techniques was compared by rapidly alternating training sessions with each chain in a parallel treatment design (Gast and Wolery 1988). A total of three comparisons were made for each participant, two with Lego $^{\circledR}{ }^{\circledR}$ structures and one with vocational structures. These comparisons were conducted sequentially; that is, for each participant, once mastery criteria were met and generalization probes were conducted for the first comparison, the second comparison began, and so on. Sessions were alternated such that no more than two sessions of either prompting procedure were conducted consecutively. Participants received approximately three training sessions per day, and sessions were conducted approximately 3 days/week. Multiple sessions in a day were separated by $10 \mathrm{~min}$ of unrelated activities.

Interobserver Agreement and Procedural Integrity

All sessions were videotaped to allow for computation of interobserver agreement and procedural integrity. A trained observer reviewed video-recorded sessions and recorded accuracy data for each step conducted in every trial of a session in $33 \%$ of sessions, distributed across participants, comparisons, and phases of the study. Percent agreement was calculated using a variation of the trial-by-trial method. For each 
trial, the total number of steps (including the training step and any mastered steps) for which there was agreement was counted. This count was divided by the number of steps in that trial and the result was multiplied by 100 . The resulting percent agreement values for each trial within a session were averaged to yield percent agreement for a session. Across all sessions and all participants, interobserver agreement ranged from 94 to $98 \%$.

Procedural integrity data also were collected by the second observer in $33 \%$ of sessions. For each trial within a session, a second observer scored whether or not (1) the correct consequence was provided for responding on the training step, (2) all prescribed prompts were followed, (3) the necessary materials were available, and (4) untrained steps were completed (they should not have been). Procedural integrity was calculated in the same way as agreement and was high, with a mean of $99 \%$.

\section{Results}

Figures 4, 5, 6, and 7 show the number of consecutive independent steps completed by each participant on the last training trial of each session. All participants acquired all structures using both hierarchies and demonstrated generalized responding in a novel setting and with a novel instructor. Both sessions to mastery and trials to mastery were calculated for each of the 12 participant-comparison combinations. MTLD resulted in fewer sessions to mastery in eight of these comparisons, and there was no difference in the number of sessions to mastery in the other four comparisons. The difference in number of sessions to mastery was small; however, there was only one comparison with more than a three-session difference between the two prompt hierarchies (Greta, comparison 1, six-session difference). Greta mastered the task in fewer sessions with MTLD in all three comparisons (see Fig. 4). Wendy and Claire mastered the construction tasks in each of the first two comparisons in one or two fewer sessions in the MTLD condition than in the MGD condition, but there was no difference in sessions-to-mastery with the vocational structures (see Figs. 5 and 6). Fred mastered all tasks with no difference between the number of sessions per condition to mastery in each of the three comparisons (see Fig. 7). Because sessions could consist of different numbers of trials, a trials-tomastery measure was analyzed. Figure 8 shows the trials to mastery for the three comparisons across all participants. Similar to the sessions-to-mastery analysis, mastery was achieved in fewer trials in the MTLD condition in 8 of the 12 comparisons. The differences, again, were small. We used a Wilcoxon signed-rank test to compare the number of trials to mastery for each of the 12 comparisons (three comparisons with four participants). Wilcoxon signed-rank test is a nonparametric test used for determining whether there is a difference between two related sets of data. In this case, we used the Wilcoxon as an alternative to a $t$ test as we did not assume the data should be normally distributed. No difference was found between MTLD and MGD $(z=1.379, p>0.05)$.

Table 1 shows the average number of errors per session with the two prompting hierarchies. Two participants (Wendy and Claire) made more errors with MGD than with MTLD. The other two participants (Greta and Fred) made more errors with MTLD than with MGD, but the average number of errors per session was similar.

\section{Discussion and Guidelines for Practitioners}

Task analysis is an evidence-based instructional strategy that is commonly used to teach various skills to learners with autism spectrum disorders and intellectual disability (Wong et al. 2013). Although there are a few published studies that may aid practitioners in the elements of designing instruction using TA, there remains a need for further investigation into the most effective and efficient strategies. One critical element in the success of a TA teaching strategy is the approach to prompting and fading. In their relatively recent study, Libby et al. (2008) found that the MTL procedure was more effective than the LTM procedure in that all skills targeted were mastered across all participants and that the MTL procedure was most efficient when paired with a constant time delay (MTLD). The current study extended Libby et al. by comparing the MTLD procedure with a simpler prompting hierarchy, MGD, which required teachers to implement fewer different types of prompts. In this study, although acquisition rate varied across participants, all participants mastered all structures with each of the prompting hierarchies used, and there was no statistically significant difference between the efficiency of the two procedures, with respect to the number of trials to mastery. With these tasks and these participants, the gradual fading of physical prompts was unnecessary. Instead, prompt fading using a simple 2-s delay to manual guidance was sufficient to transfer stimulus control from teacher-delivered prompting to the desired stimuli, the required materials, and the initial instruction to complete the task. Given a choice between two effective teaching procedures, practitioners may consider the relative ease of implementation. In this way, the MGD procedure may be superior to the MTLD.

Prompting hierarchies that are easier for instructors to implement may be beneficial to practitioners and learners for several reasons. First, it is likely that the procedure would be implemented more frequently if the instructor were confident and efficient with the procedure. With MGD, the only procedural element that changes as prompts are reduced is the delay to the prompt; the level of manual guidance remains constant until the prompt is withheld. Teaching using a TA is simpler 
Fig. 4 Independent steps completed for both leisure comparisons and the vocational task for Greta. Symbols show the last step performed independently for the participant in the last trial of each session during the MTLD condition (filled circles) and the MGD condition (open squares). The gray symbols show the number of independent steps during generalization probes following MTLD (circle) and MGD (square) training
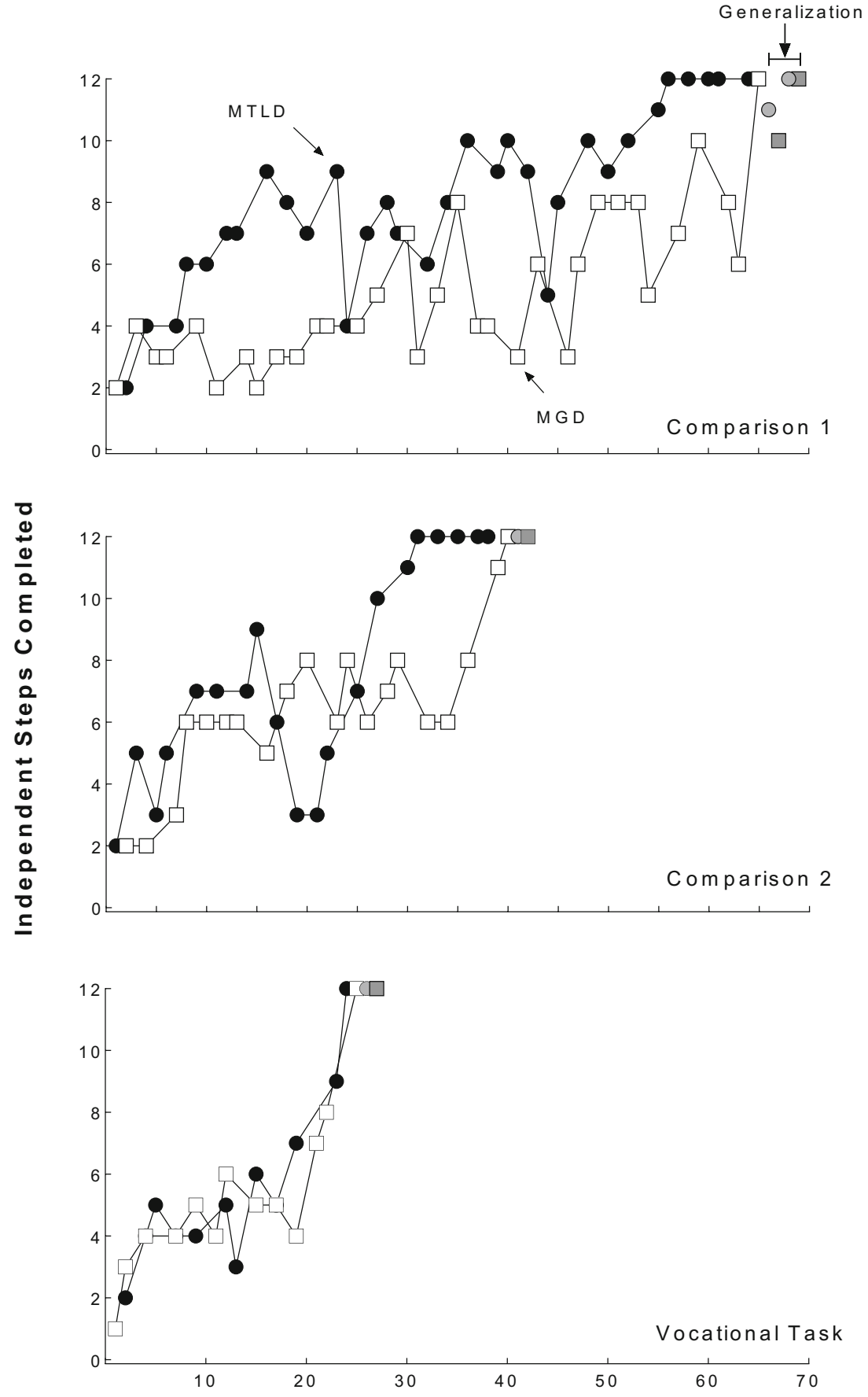

Session and may be easier with MGD, especially for those with less advanced training or experience. Social validity, including the desirability of an intervention, is a moderator of the impact on that intervention on the well-being of the consumer (Foster and Mash 1999; Schwartz and Baer 1991). Additionally, it likely would be less effortful to train new teachers to properly implement teaching procedures that are simpler. This could lead to a savings in resources when it comes to training and verifying competency in direct-care staff. Future research could compare the relative social validity of the MGD procedure with more complex procedures, as well as the minimal resources required for training staff to competency.

Second, it is likely that procedural integrity will be greatest with procedures that are relatively easy to implement. For example, a teaching procedure that does not require gradual 
Fig. 5 Independent steps completed for both leisure comparisons and the vocational task for Wendy. Symbols show the last step performed independently for the participant in the last trial of each session during the MTLD condition (filled circles) and the MGD condition (open squares).

The gray symbols show the number of independent steps during generalization probes following MTLD (circle) and MGD (square) training
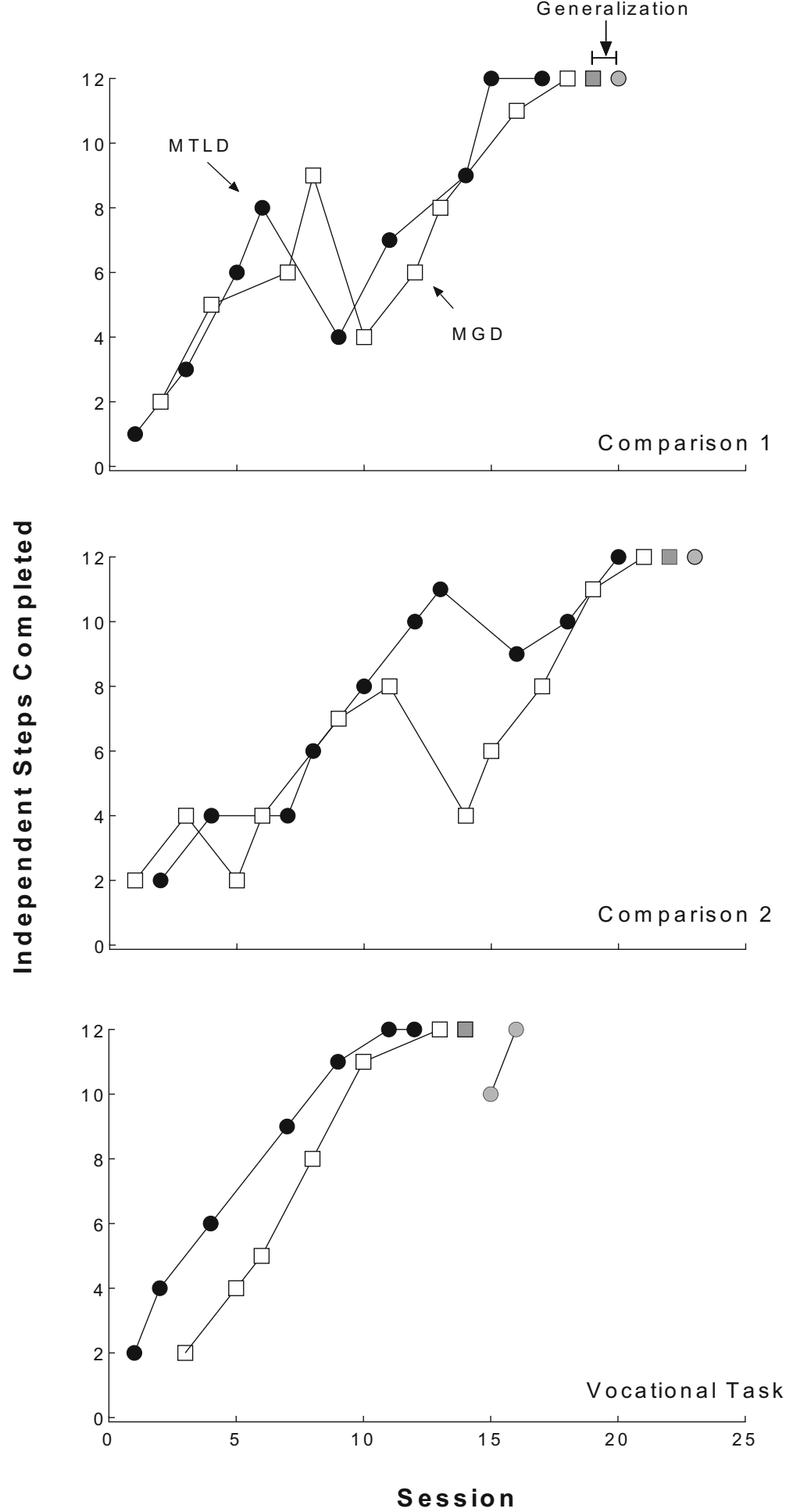

W endy fading of physical prompts would likely be easier for instructors to implement in comparison with one that does require such fading. Enhanced procedural integrity is associated with better intervention outcomes (Fryling et al. 2012). Ensuring a high degree of procedural integrity can be especially challenging in situations where several individuals serve as teachers for a given learner, as is typically the case in special education classrooms and group homes. In the current study, there were no differences in procedural integrity across conditions; future 
Fig. 6 Independent steps completed for both leisure comparisons and the vocational task for Claire. Symbols show the last step performed independently for the participant in the last trial of each session during the MTLD condition (filled circles) and the MGD condition (open squares). The gray symbols show the number of independent steps during generalization probes following MTLD (circle) and MGD (square) training. The asterisk denotes an occasion wherein mastery criteria were met by accurate performance on a probe trial

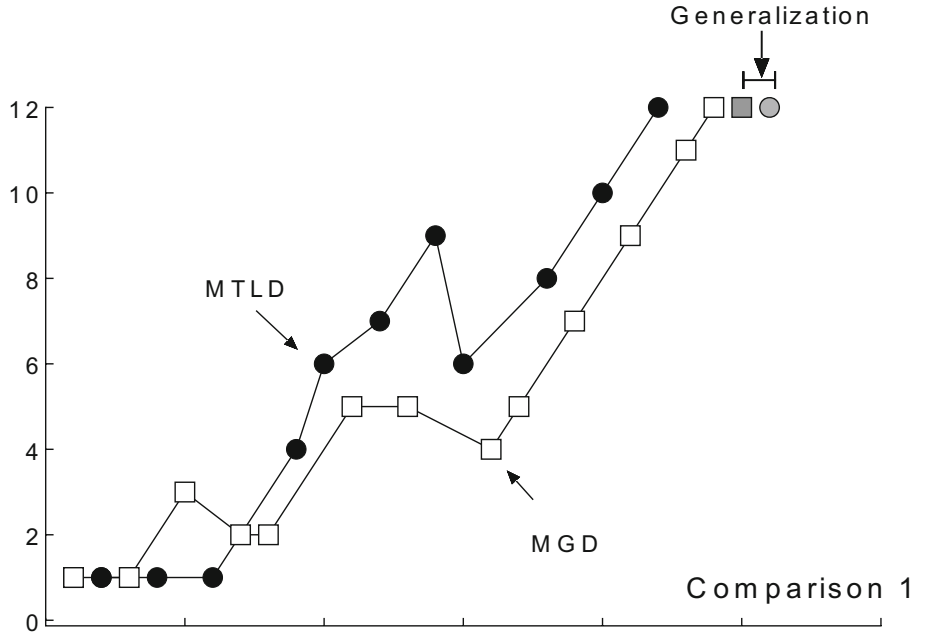

0
0
00
$\frac{0}{0}$
$\varepsilon$
0
0
0
0
0
0
0
\pm
0
0
0
0
0
0
0
$\leq$
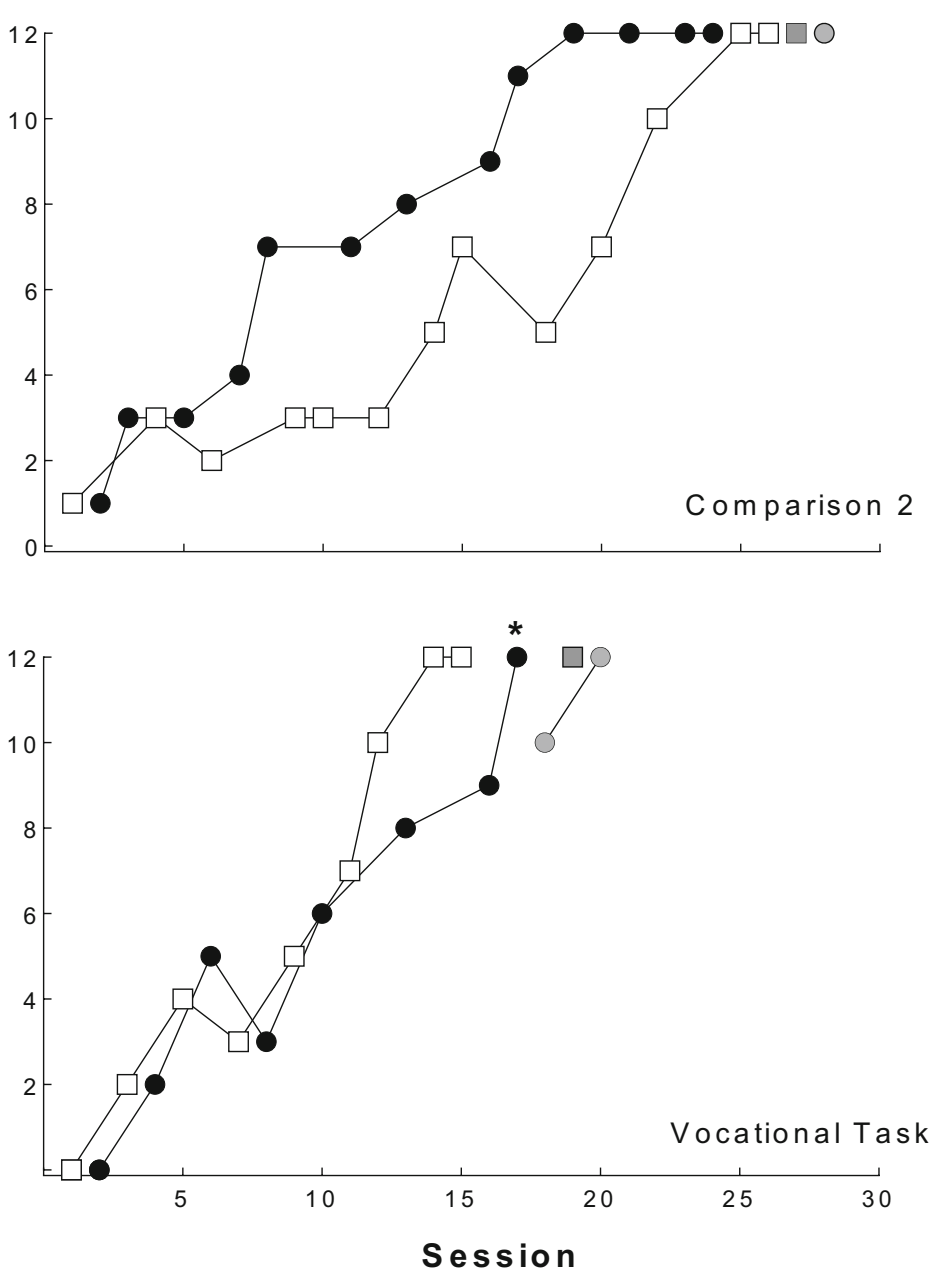

Claire studies could be designed to measure procedural integrity with different prompting procedures in the applied setting or across a wider variety of tasks, including selfcare and communication skills.
In addition to providing comparisons of procedural integrity and other implementation variables, future research could expand the scope of evidence available to practitioners as they make decisions regarding teaching procedures. In the study 
Fig. 7 Independent steps completed for both leisure comparisons and the vocational task for Fred. Symbols show the last step performed independently for the participant in the last trial of each session during the MTLD condition (filled circles) and the MGD condition (open squares).

The gray symbols show the number of independent steps during generalization probes following MTLD (circle) and MGD (square) training. The asterisk denotes an occasion wherein mastery criteria were met by accurate performance on a probe trial

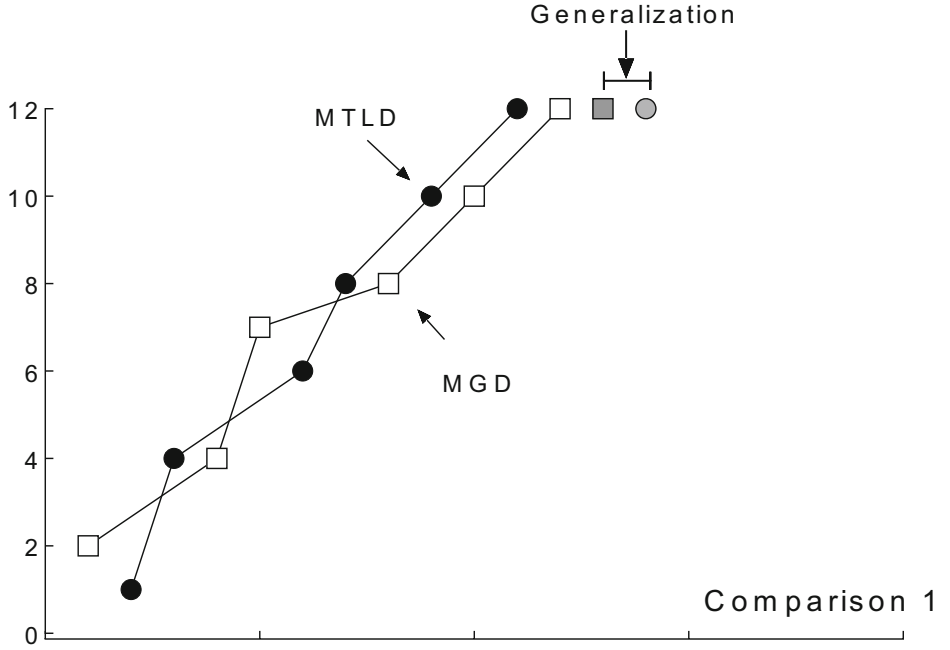

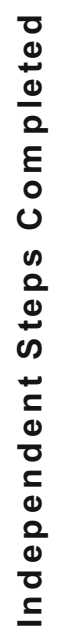

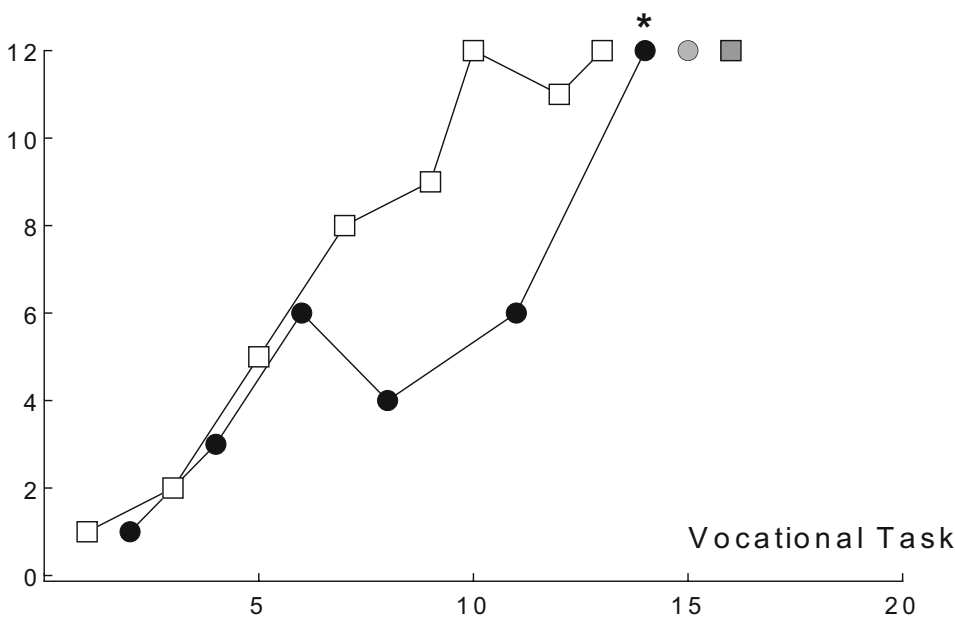

Session

Fred

presented here, participants learned to complete various assembly and construction tasks. Task analyses are used to teach a wide range of performances, from domestic (Cuvo et al.
1992; Walls et al. 1981) and personal hygiene skills (Horner and Keiltiz 1975; Stokes et al. 2004) to communication (Sigafoos et al. 1994) and leisure and athletic skills (Cuvo 
Fig. 8 Trials to mastery in MTLD (black bars) and MGD (white bars) for both leisure comparisons and the vocational task across all participants
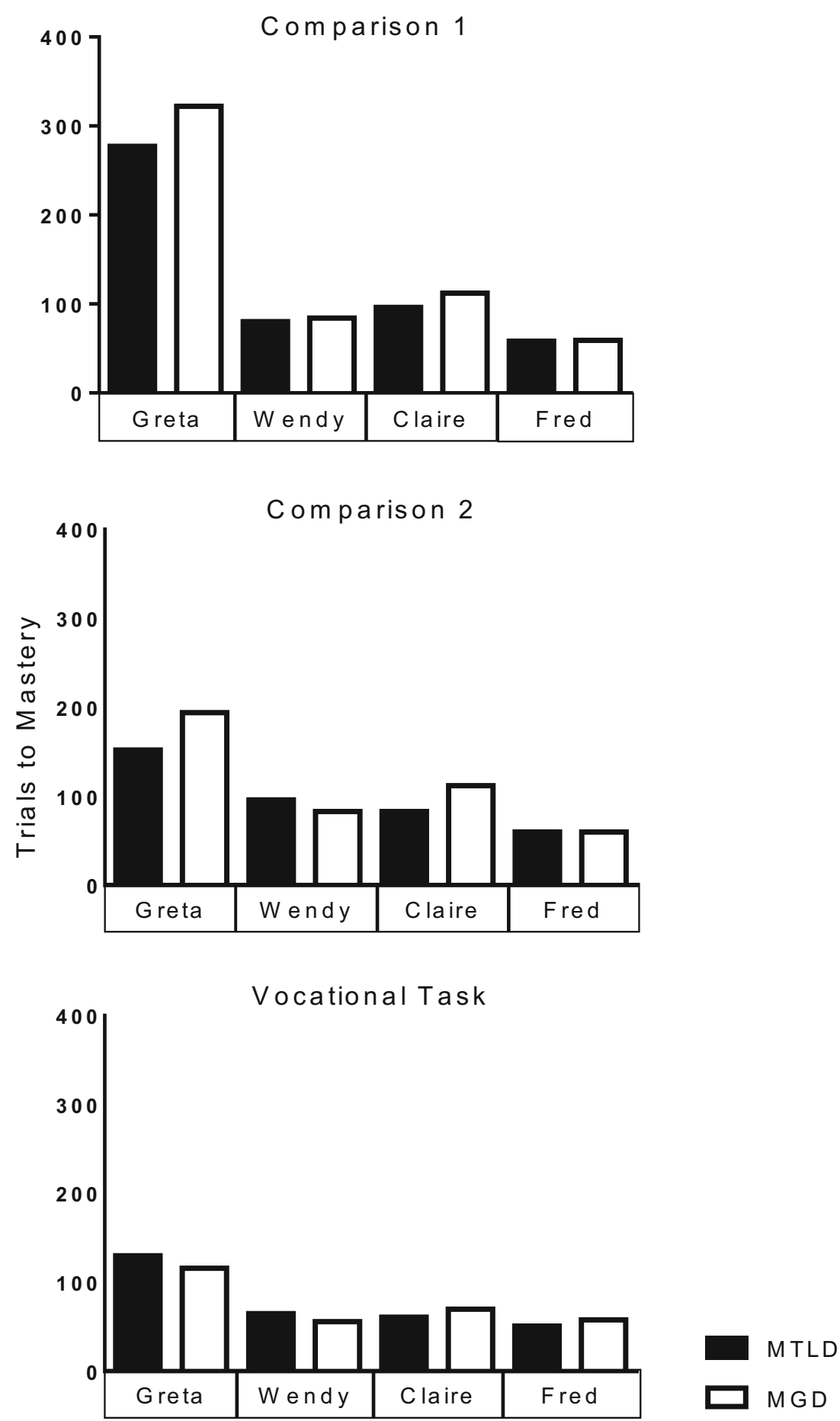

Table 1 Average number of errors per session for each participant

\begin{tabular}{lll}
\hline \multirow{2}{*}{ Participant } & \multicolumn{2}{l}{ Average errors per session } \\
\cline { 2 - 3 } & MTLD & MGD \\
\hline Greta & 3.81 & 3.58 \\
Wendy & 1.79 & 2.30 \\
Claire & 2.46 & 3.03 \\
Fred & 2.00 & 1.89 \\
\hline
\end{tabular}

et al. 1983; Jerome et al. 2007). In future research, comparisons of teaching strategies should be conducted across a range of skills. It is possible that differences between tasks would moderate the effects of the teaching procedures. For example, participants seated at a table completed the tasks in this study, but some tasks require the learner to move between environments (e.g., the standing long jump and 50-yard dash in Cuvo et al. 1983). The extent to which the prompt fading strategies used in the current study would yield similar results across 
different settings and skills is unknown, and practitioners could benefit from continued research that compares these teaching procedures across skills, settings, and learners with varied characteristics.

Comparisons of efficacy and ease-of-implementation also should continue with prompting modalities other than manual guidance, such as modeling (including video modeling) and verbal prompting. These comparisons should be conducted with learners of various skills levels and across settings and types of tasks. It would be useful to know how the effects of teaching procedures and prompting modalities are moderated by student characteristics. It is possible that some learner characteristics such as their existing repertoire and their tendency to respond to (or away from) certain stimuli could moderate effects of these teaching procedures. In this study, the participants had a history of learning with manual guidance. For these learners, manual guidance did not lead to challenging behavior, and it did not appear that the prompts were aversive. In particular, two of the participants in this study, Wendy and Claire, made more errors with the MGD procedure than with the MTLD. These errors did not appear to interfere with acquisition, but had they been students for whom manual guidance were aversive, MTLD, wherein the level of manual guidance is reduced following successful performance may have been preferred over the MGD procedure. In such a case where MGD may be contraindicated, the more complicated MTLD procedure could be considered a remedial procedure, whereby the more complex system of fading would be used as needed.

It should be noted that manual guidance may be problematic in some situations, regardless of how prompting is faded. Some learners will resist manual guidance, and for others, it will be a positive reinforcer. In the former case, escape or avoidance behavior may occur, and in the latter, the learner may work to maximize manual guidance. Additionally, in some settings, such as in the community, the use of manual guidance may be stigmatizing for the learner or uncomfortable for others. In each of these cases, a prompting modality that does not include manual guidance should be considered. Comparisons of effects of different prompting modalities and effects of variations of these prompting modalities could contribute to the evidence base to guide practitioners to select optimal teaching strategies for a given learner.

When teaching using TA, there are many variables to consider; the more practitioners know about which of these are most important, the more effective they can be as they design interventions and develop and implement trainings for direct-care staff. Component analysis of behavioral interventions is commonly called for as a strategy for determining what elements of an intervention are most important. This is especially important with complex or difficult-to-implement procedures. Allen and Warzak (2000) suggest further in their work on parental adherence to treatment plans that the best outcome for consumers may be obtained with procedures that result in good outcomes and that are most likely to be adopted by persons providing care to the consumer; in some cases, such procedures may be more desirable than those that result in the best outcomes in the laboratory. The current study examined one component of one of a teaching procedure that previously had been shown to be effective and found the gradual fading of physical prompts to be an unnecessary component of MTLD. We look forward to consuming more reports that inform practitioners as to the necessary and optimal components of procedures for teaching our consumer skills that will maximize their independence and access to reinforcement. In the meantime, in the interest of providing effective teaching procedures that have the greatest likelihood of proper and frequent implementation, we recommend the use of the MGD prompt fading hierarchy though we suggest that best practice involves identifying the most effective teaching procedure for each individual.

Acknowledgments This research was supported by the New England Center for Children and is based on the thesis completed by Julie McKay in partial fulfillment of requirements for a Master of Science degree from Northeastern University.

Special thanks to Shawn Kenyon for his contributions. This manuscript is dedicated to Myrna Libby and all the meaningful research she contributed to the field of applied behavior analysis. This project was a product of her previous research on prompting and her ideas to improve teaching skills to individuals with intellectual disabilities.

\section{References}

Allen, K. D., \& Warzak, W. J. (2000). The problem of parental nonadherence in clinical behavior analysis: effective treatment is not enough. Journal of Applied Behavior Analysis, 33, 373-391.

Axelrod, S. (1991). The problem: American education. The solution: use behavior analytic technology. Education and Treatment of Children, 1, 275-282.

Axelrod, S. (1992). Disseminating an effective educational technology. Journal of Applied Behavior Analysis, 25, 31-35.

Bancroft, S. L., Weiss, J. S., Libby, M. E., \& Ahearn, W. H. (2011). A comparison of procedural variations in teaching behavior chains: manual guidance, trainer completion, and no completion of untrained steps. Journal of Applied Behavior Analysis, 44, 559-569.

Billingsley, F. F., \& Romer, L. T. (1983). Response prompting and the transfer of stimulus control: methods, research and a conceptual framework. Journal of the Association for the Severely Handicapped, 8, 3-12.

Carr, E. G., \& Durand, V. M. (1985). Reducing behavior problems through functional communication training. Journal of Applied Behavior Analysis, 18, 111-126.

Cuvo, A. J., Davis, P. K., O’Reilly, M. F., Mooney, B. M., \& Crowley, R. (1992). Promoting stimulus control with textual prompts and performance feedback for persons with mild disabilities. Journal of Applied Behavior Analysis, 25, 477-489.

Cuvo, A. J., Ellis, P. J., Wisotzek, I. E., Davis, P. J., Schilling, D., \& Bechtal, R. (1983). Teaching athletic skills to students who are mentally retarded. Journal of the Association for Persons with Severe Handicaps, 8, 72-81. 
Demchak, M. (1990). Response prompting and fading methods: a review. American Journal of Mental Retardation, 94, 603-615.

Detrich, R. (1999). Increasing treatment fidelity by matching interventions to contextual variables within the educational setting. School Psychology Review, 28, 608-620.

Etzel, B. C., \& LeBlanc, J. M. (1979). The simplest treatment alternative: the law of parsimony applied to choosing appropriate instructional control and errorless-learning procedures for the difficult-to-teach child. Journal of Autism and Developmental Disorders, 9, 361-382.

Fisher, W. W., Piazza, C. C., Bowman, L. G., Hagopian, L. P., Owens, J. C., \& Slevin, I. (1992). A comparison of two approaches for identifying reinforcers for persons with severe and profound disabilities. Journal of Applied Behavior Analysis, 25, 491-498.

Foster, S. L., \& Mash, E. J. (1999). Assessing social validity in clinical treatment research: issues and procedures. Journal of Consulting and Clinical Psychology, 67, 308-319. doi:10.1037/0022-006X.67. 3.308 .

Fryling, M. J., Wallace, M. D., \& Yassine, J. N. (2012). Impact of treatment integrity on intervention effectiveness. Journal of Applied Behavior Analysis, 45, 449-453. doi:10.1901/jaba.2012. 45-449.

Gast, D. L., \& Wolery, M. (1988). Parallel treatments design: a nested single subject design for comparing instructional procedures. Education and Treatment of Children, 11, 270-285.

Grow, L., Carr, J. E., Gunby, K. V., Charania, S. M., Gonsalves, L., Ktaech, I. A., \& Kisamore, A. N. (2009). Deviations from prescribed prompting procedures: implications for treatment integrity. Journal of Behavioral Education, 18, 142-156.

Horner, R. D., \& Keilitz, I. (1975). Training mentally retarded adolescents to brush their teeth. Journal of Applied Behavior Analysis, 8, 301-309.

Jerome, J., Frantino, E. P., \& Sturmey, P. (2007). The effects of errorless learning and backward chaining on the acquisition of internet skills in adults with developmental disabilities. Journal of Applied Behavior Analysis, 40, 185-189.
Libby, M. E., Weiss, J. S., Bancroft, S., \& Ahearn, W. H. (2008). A comparison of most-to-least and least-to-most prompting on the acquisition of solitary play skills. Behavior Analysis in Practice, 1, $37-43$.

Mansfield, R., Dudley, L., DeGregory, K., \& Foster, K. (2011). U.S. Patent No. 7,974,569 B2. Washington, DC: U.S. Patent and Trademark Office.

Schwartz, I. S., \& Baer, D. M. (1991). Social validity assessments: is current practice state of the art? Journal of Applied Behavior Analysis, 24, 189-204. doi:10.1901/jaba.1991.24-189.

Sigafoos, J., Kerr, M., Roberts, D., \& Couzens, D. (1994). Increasing opportunities for requesting in classrooms serving children with developmental disabilities. Journal of Applied Behavior Analysis, 24, 631-645.

Spooner, F., \& Spooner, D. (1984). A review of chaining techniques: implications for future research and practice. Education and Training of the Mentally Retarded, 19, 114-124.

Stokes, J. V., Cameron, J. J., Dorsey, M. F., \& Fleming, E. (2004). Task analysis, correspondence training, and general case instruction for teaching personal hygiene skills. Behavioral Interventions, 19, 121135.

Walls, R. T., Crist, K., Sienicki, D. A., \& Grant, L. (1981). Prompting sequences in teaching independent living skills. Mental Retardation, $19,243-246$.

Wolery, M., \& Gast, D. L. (1984). Effective and efficient procedures for the transfer of stimulus control. Topics in Early Childhood Special Education, 4, 52-77.

Wong, C., Odom, S. L., Hume, K. Cox, A. W., Fettig, A., Kucharczyk, S., ... Schultz, T. R. (2013). Evidence-based practices for children, youth, and young adults with autism spectrum disorder. Chapel Hill: The University of North Carolina, Frank Porter Graham Child Development Institute, Autism Evidence-Based Practice Review Group. Retrieved from http://fpg.unc.edu/sites/fpg.unc. edu/files/resources/reports-and-policy-briefs/2014-EBP-Report.pdf. 